\title{
OPTIMIZATION OF A MULTISTOREY-BUILDING BY OPTIMUM POSITIONING OF SHEAR WALL
}

\author{
Sejal Bhagat \\ Department of Civil Engineering, Sarvajanik College of Engineering and Technology,Surat-395001,Gujarat,India.
}

\begin{abstract}
The shear wall is a structural element which is used to resist earthquake forces. These wall will consumptives shear forces \& will prevent changing location-position of construction \& consequently destruction. On other hand, shear wall arrangement must be absolutely accurate, if not, we will find negative effect instead. For example if the shear walls make an increase distance between mass centre and hardness centre, we cannot expect a good tensional behavior from the structure. In case of mass centre and hardness centre coincide with each other, at that time the distance of shear wall from the mass centre also plays an important role in the shear contribution of the shear wall. The bending moment, shear force, torsion, axial force contribution by rest of the structural element and the ultimate design of all the structural components also affected by that. A study has been carried out to determine the optimum Structural configuration of a multistory building by changing the shear wall locations radically. Four different cases of shear wall position for a 10 storey residential building with keeping zero eccentricity between mass centre and hardness centre have been analyzed and designed as a space frame system by computer application software, subjected to lateral and gravity loading in accordance with IS provisions.
\end{abstract}

Keywords: Shear walls, Lateral loading, Zero eccentricity, Stresses, Design configuration.

\section{INTRODUCTION}

Constructing the Shear wall in tall, medium and even short buildings will reinforce the significantly and either more economic than the bending frames. By the Shear walls, we can control the side bending of the structure, much better than other elements like closed frames and certainly the shear walls are more flexible than them. However, on many occasions the design has to be based on the off center position of the Lift and stair case walls with respect to the center of mass. The design in these cases results into an excessive stresses in most of the structural members, unwanted torsional moments and sways. Design by coinciding Stiffness center and mass of the building is the ideal for a structure. In this case there is no eccentricity, but as per IS 1893(1):2002 the minimum eccentricity is to be considered. The lateral force in a wall due to rotational moment is given by,

$$
\text { Fir }=\frac{\text { kiri }}{\sum \text { kiri }^{2}}\left(\mathrm{Fe}_{\mathrm{d}}\right)
$$

Where, $\mathrm{ki}=$ Stiffness of Shear wall "i"

ri $=$ Radial distance of shear wall "i"

$\mathrm{F}=$ Design Shear force

ed $=$ Design eccentricity

From the above equation, it is observed that the distance of any shear wall from the centre of stiffness increases, the Shear generated in the Shear wall is decreased. The distance of Shear wall from the Centre of Stiffness is also an important Criteria for the Stresses generated in the Structural members and overall behavior of the whole structure. 


\subsection{Methodology}

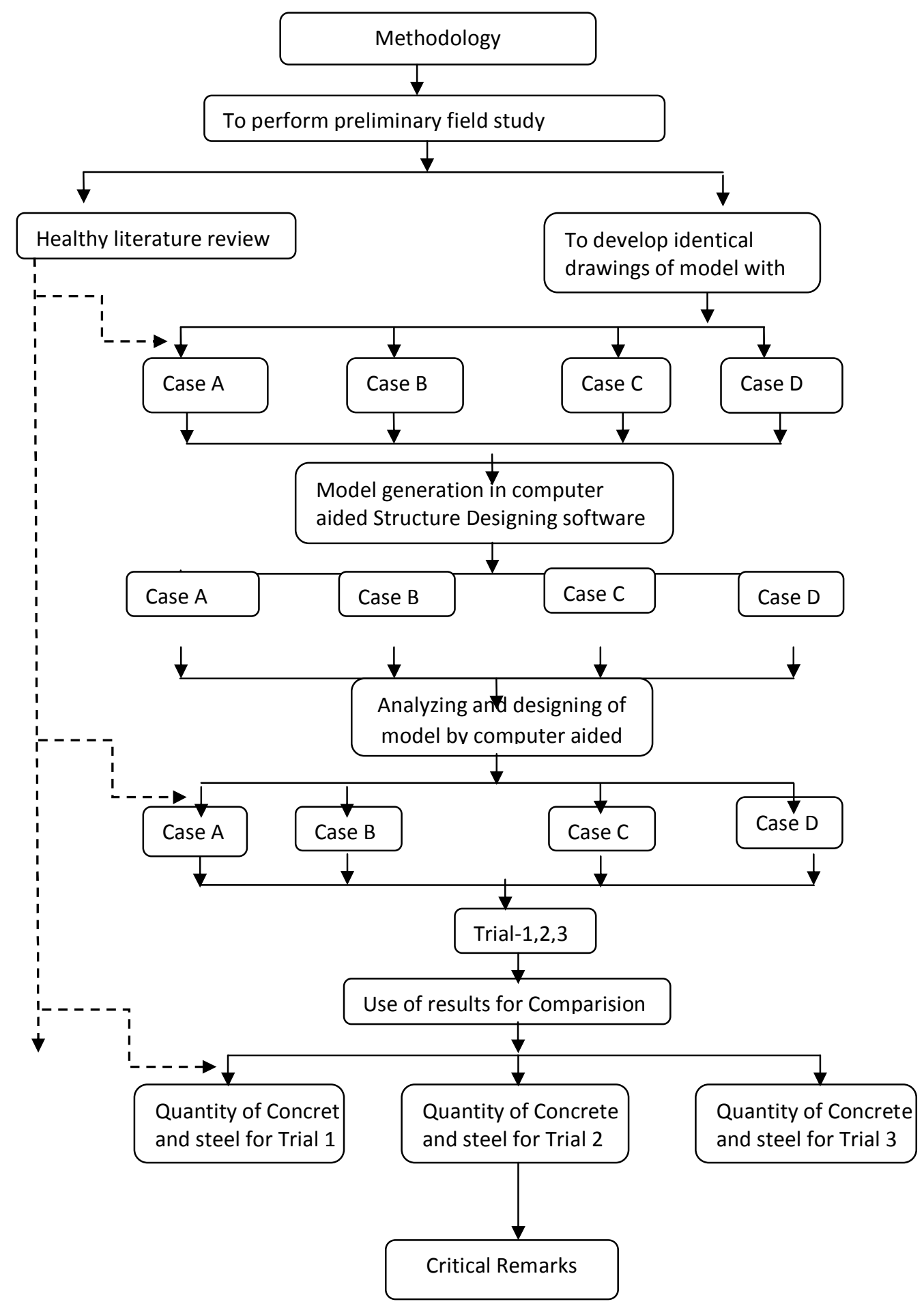




\section{Analytical Study}

\subsection{Problem Statement}

\subsubsection{General}

The Building will be used for residential Purpose. So that there are $115 \mathrm{~mm}$ thick interior walls and $230 \mathrm{~mm}$ exterior walls are considered.

$>$ For simplicity in analysis, no balconies used in the building.

$>$ At ground floor, slabs are not provided and the floor will directly rest on ground.

$>$ The main beams rest centrally on columns to avoid local eccentricity.

$>$ Center line dimensions are followed for analysis and design.

\subsubsection{Data of the Example}

A typical building $(\mathrm{G}+8)$ having three various position of shear wall and one without shear wall having following data

Floor to Floor height

$=3000 \mathrm{~mm}$

Height of Plinth

$=450 \mathrm{~mm}$ above ground

level.

Depth of Foundation

level.

External Walls

Internal Walls

\subsubsection{Imposed Loads}

Roof: Roof Finish

Live Load

Floor: Floor Finish

Live Load

\subsubsection{Earthquake Load}

EQ load generation method Method

Seismic Zone

Soil Type

Percentage Damping

Modal Combination method

$$
\begin{aligned}
& =230 \mathrm{~mm} \\
& =115 \mathrm{~mm}
\end{aligned}
$$

\subsubsection{Materials}

Concrete

Steel: Main \& Secondary

Unit Weight of Concrete

Unit Weight of Bricks Masonry

Design Basis:

based on IS: 456-2000

$$
=1.5 \mathrm{KN} / \mathrm{m} 2
$$

$=$ Variable parameter

$=1.0 \mathrm{KN} / \mathrm{m} 2$

$=$ Variable parameter

$=$ Response Spectrum

$=$ Zone 3

$=$ Medium Soil

$=5 \%$

$=\mathrm{SRSS}$

$=\mathrm{M} 20$,

$=\mathrm{Fe} 415$

$=25 \mathrm{KN} / \mathrm{m} 2$

$=19 \mathrm{KN} / \mathrm{m} 2$

=Limit State Method

\subsubsection{Typical Drawing of Each Case}

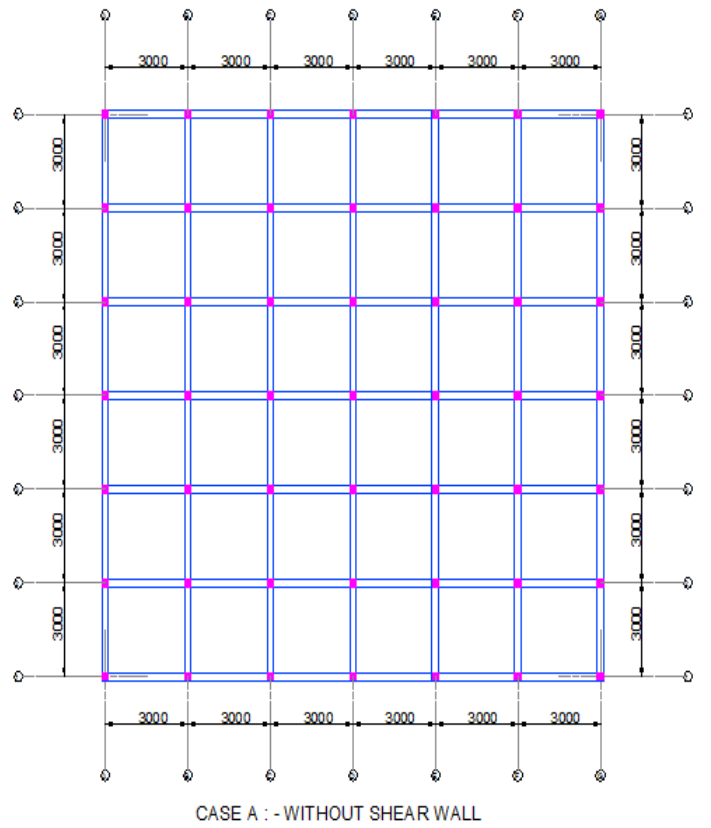

Fig 1 :- Location of shear wall in case A

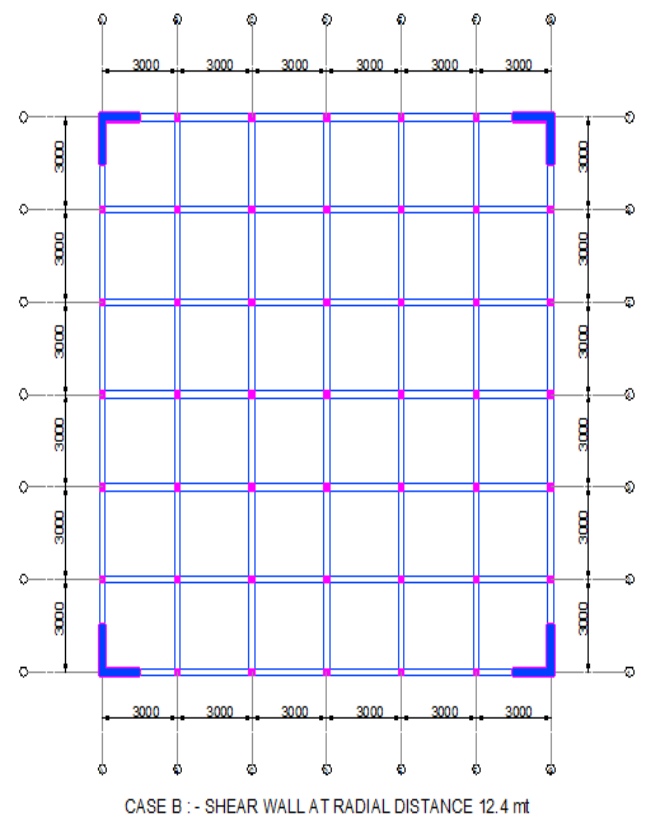

Fig2 :- Location of shear wall in case B 


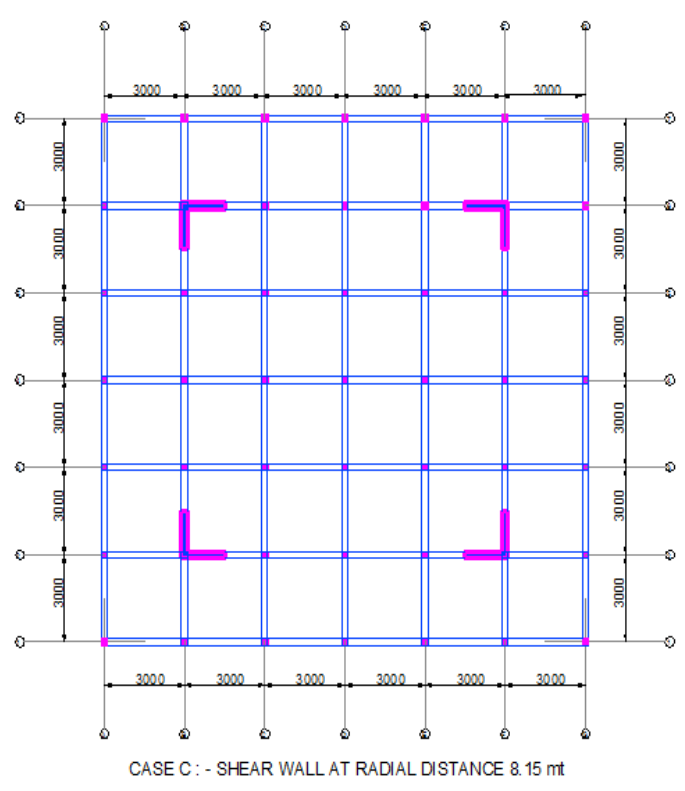

Fig 3:- Location of shear wall in case $\mathrm{C}$

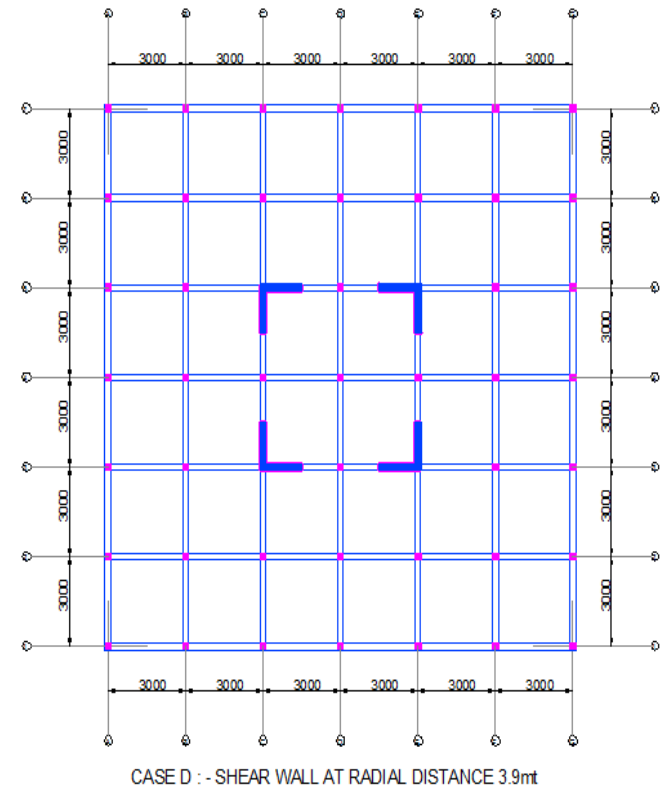

Figure 4:- Location of shear wall in case D

\subsubsection{Live Load}

Live loads are also called the superimposed loads and include all the moving or variable loads, due to people or occupants, their furniture, temporary stores, machinery etc. Live loads on floors shall compromise of all loads other than the dead loads. The various live loads acting on the different floors are given in IS 875: 1998

\subsubsection{Earthquake Load}

EQ load acts on the structure during earthquake. It will act horizontally on the structure. It is also called as seismic force.

\subsubsection{Methods of Analysis}

There are three methods for building analysis which have been used for the analysis of reinforced concrete structure viz. Plane grid method, Plane frame method, and Space frame method.

Space frame method is the most accurate and desirable method for analysis. This method is difficult to adopt for manual calculations, but is most suitable for computer-aided analysis. In the Space frame method, the stiffness of columns is taken into account for analysis. Beams are designed as continuous beams with fixity at end supports. Columns will be designed for axial load and moments in $\mathrm{X}$ and $\mathrm{Y}$ directions. Footing shall also be designed for biaxial bending. 


\subsubsection{Design Philosophies}

There are three design philosophies which have been used for the design of reinforced concrete structure viz. working stress method, Ultimate load method, Limit sate method.

At present the IS 456-2000 recommends the use of limit state method of design. However it has also retained the working stress method of design. A brief introduction about limit state method is represented below.

\subsubsection{Limit State Method (IS 456-2000)}

It is based on the concept as to achieve an acceptable probability that the structure will not become unserviceable in its lifetime. Hence this method is based on its philosophy that the structure should be able to withstand safely the working load throughout its life and also satisfy the serviceability requirement. In the design the following limit states are examined:

\section{Limit State of Collapse:}

It corresponds to the maximum load carrying capacities and it violation implies failure but do not mean complete collapse. This limit state corresponds to: Flexure, Compression, Shear and torsion.

\section{Limit State of Serviceability:}

It corresponds to the development of excessive deformation. This state corresponds to Defection on cracking and vibration.

\subsubsection{Design of Building Components}

A brief description about the various components of the building (Super and Substructure) along with the method of analysis and design is presented in following articles.

\subsubsection{Slabs}

Slabs may be classified a one way slabs or two-way slabs based up on the aspect ratio. When the aspect ratio (ly/lx) $>2$. It is designed as one-way slab. However it is designed as two way slab when aspect ratio is $\leq 2$.

$>$ One way slab are designed as a beam considering one meter width of slab.

$>$ Two way slabs are further classified into nine types as given in IS 456:2000 based on boundary conditions.

$>$ The two way slabs are divided in middle strips and edge strips and designed accordingly.

\subsubsection{Beams}

These are the basically flexural member on which the slab rest. The beam is supported on columns to which they transmit the loads. Beams can have square, rectangular or flanged ( $\mathrm{T}$ or
L-shaped) cross-sections. With respect to the reinforcement provided. Beams can be singly reinforced or doubly reinforced.

\subsubsection{Column}

These are the vertical skeletal structural elements which may be rectangular, square, circular etc. in their cross-sectional shapes. The size of the section is governed by effective length of the column and loads acting on it, which in turn depend on the type floor system, spacing of columns, number of storey etc. The column is generally designed to resist axial compression combined with uni-axial or Bi-axial bending moments that are induced by frame action. It is also advisable to reduce the unsupported length of the columns by providing appropriate tie beams-otherwise they may have to be designed as slender columns.

\subsubsection{Footing}

These are the elements provided at the ground level to transfer the load of column to the soil. The design of footing is carried out with flexure, one way shear and two way shear consideration. The area of footing is provided based on the soil bearing capacity.

\subsubsection{Shear wall}

Shear walls are vertical elements in the lateral-force-resisting system that transmits lateral forces from the diaphragm above to the diaphragm below, or to the foundation. Shear walls may also be bearing walls in the gravity-load system, or they may be components in a dual system framed so as to resist only lateral loads.

Walls may be subjected to both vertical (gravity) and horizontal (wind or earthquake) forces. The horizontal forces are both in plane and out-of-plane. When considered under their in-plane loads, walls are called shear walls; when considered under their out-of-plane loads, they are called normal walls. Walls will be designed to withstand all vertical loads and horizontal forces, both parallel to and normal to the flat surface, with due allowance for the effect of any eccentric loading or overturning forces generated.

In this analyasis all the elements are analyzed and designed by space frame method and limit state method respectively by use of computer aided software "STRUDS 2008"

\subsection{Calculation of R.C.C. Design}

\subsubsection{Analysis Process}

Methods of analysis for a multistory structure are:

1) Approximate methods

$>$ Substitute frame method

$>$ Portal frame method 
2) Computer Methods

$$
\text { cantilever frame method }
$$

$>$ Matrix methods - Flexibility Matrix , Stiffness Matrix methods

$>$ Finite Element methods

$>$ Finite difference methods

Among all the above methods most accurate method is computer aided software. For computer analysis and design of structures various software are available in the market like STRUDS 2008, STADD PRO V8i, ETABS, and ANSYS etc. The analysis of the whole project is done by computer aided software "STRUDS 2008".The modeling, analysis and design process for the structure is done by this software in three modes. The procedure of analysis in STRUD 2008 is as follows:

\subsubsection{Pre-Processing Mode}

This process consists of the following procedure:

1) File opening and preferences setting.

2) Creation of various types of slabs with varying supporting conditions.

3) Locating Columns.

4) Specifying Boundary conditions.

5) Enter material and Section properties

6) Generating walls in the floor plan.

7) Observing Entities.

8) Attaching external loads.

9) Copying the floor plans.

10) Editing the floor plans.

11) Analyzing the building structure.

\subsubsection{Post Processing Mode}

Analysis report is obtained in this mode. Report of post processing mode can be generated are

a) Elemental result

1) Shear force

Bending moment

Axial force

2) Torsion b) Nodal result

1) Deflection

2) Reaction

\subsubsection{Design Process}

Design of each components of multistoried structure has been done by using computer aided software STRUD 2008 in the following way,

a) Setting of Design parameters for
1) Slab
2) Beam
3) Column
4) Footing
5) Shear wall

b) Design of each components one by one

c) Design data reports

1) Design schedules for each component

\subsubsection{Quantity Estimation}

In this software the quantity of each R.C.C. components has been provided with quantity of concrete and quantity of steel separately.

Quantity reports of structure has been obtain by software for following components

1) Slab quantity

2) Beam quantity

3) Column quantity

4) Footing quantity

\section{Result and Discussion}

\subsection{Results}

\subsubsection{Trial:-1}

$>$ Live Load $=2 \mathrm{KN} / \mathrm{m} 2$

$>$ Preliminary Beam Size $=230$ x $450 \mathrm{~mm}$

\subsubsection{Beam}

Table 1: Quantity of concrete in beam (trial 1)

\begin{tabular}{|l|l|l|l|l|}
\hline QUANTITY OF CONCRETE (FLOOR WISE) \\
\hline FLOOR NO. & CASE A & CASE B & CASE C & CASE D \\
\hline 1 & 26.082 & 25.081 & 25.185 & 25.288 \\
\hline 2 & 26.082 & 25.702 & 25.737 & 26.030 \\
\hline 3 & 26.082 & 25.116 & 24.840 & 24.961 \\
\hline 4 & 26.082 & 24.978 & 24.840 & 24.909 \\
\hline 5 & 26.082 & 24.840 & 24.840 & 24.909 \\
\hline 6 & 26.082 & 24.840 & 24.840 & 24.840 \\
\hline 7 & 26.082 & 24.840 & 24.840 & 24.840 \\
\hline 8 & 26.082 & 24.840 & 24.840 & 24.840 \\
\hline
\end{tabular}




\begin{tabular}{|l|l|l|l|l|}
\hline 9 & 26.082 & 24.840 & 24.840 & 24.840 \\
\hline 10 & 26.082 & 24.840 & 24.840 & 24.840 \\
\hline TOTAL(m3) & $\mathbf{2 6 0 . 8 2}$ & $\mathbf{2 4 9 . 9 1 7}$ & $\mathbf{2 4 9 . 6 4 2}$ & $\mathbf{2 5 0 . 2 9 7}$ \\
\hline
\end{tabular}

Table 2: Quantity of steel in beam (trial 1)

\begin{tabular}{|l|l|l|l|l|}
\hline QUANTITY OF STEEL (FLOOR WISE) \\
\hline FLOOR NO. & CASE A & CASE B & CASE C & CASE D \\
\hline 1 & 2772.227 & 2675.430 & 3032.986 & 3175.756 \\
\hline 2 & 2930.800 & 3232.510 & 3825.537 & 3962.128 \\
\hline 3 & 2102.556 & 2332.150 & 2675.695 & 2711.517 \\
\hline 4 & 2033.926 & 2367.998 & 2801.699 & 2750.90 \\
\hline 5 & 1967.653 & 2382.478 & 2825.914 & 2719.660 \\
\hline 6 & 1930.262 & 2341.676 & 2814.228 & 2659.814 \\
\hline 7 & 1927.452 & 2310.357 & 2750.929 & 2579.280 \\
\hline 8 & 1920.258 & 2279.415 & 2668.587 & 2489.191 \\
\hline 9 & 1920.258 & 2205.625 & 2637.418 & 2411.293 \\
\hline 10 & 1920.258 & 2060.936 & 2469.929 & 2343.405 \\
\hline TOTAL(kg) & $\mathbf{2 1 4 2 5 . 6 9 3}$ & $\mathbf{2 4 1 8 8 . 5 7 5}$ & $\mathbf{2 8 5 0 2 . 9 2 2}$ & $\mathbf{2 7 8 7 4 . 9 4 4}$ \\
\hline
\end{tabular}

\subsubsection{Column}

Table3: Quantity of concrete in column (trial 1)

\begin{tabular}{|l|l|l|l|l|}
\hline QUANTITY OF CONCRETE (FLOOR WISE) \\
\hline FLOOR NO. & CASE A & CASE B & CASE C & CASE D \\
\hline 1 & 13.296 & 10.941 & 10.630 & 10.706 \\
\hline 2 & 15.642 & 14.336 & 14.336 & 13.466 \\
\hline 3 & 13.814 & 11.894 & 11.041 & 10.201 \\
\hline 4 & 11.219 & 9.972 & 9.360 & 9.207 \\
\hline 5 & 10.607 & 9.054 & 7.830 & 8.059 \\
\hline 6 & 8.159 & 7.525 & 7.141 & 7.414 \\
\hline 7 & 7.776 & 7.776 & 7.141 & 7.414 \\
\hline 8 & 7.776 & 7.776 & 7.141 & 7.414 \\
\hline 9 & 7.776 & 7.776 & 7.141 & 7.414 \\
\hline 10 & 7.776 & 7.776 & 7.141 & 7.414 \\
\hline TOTAL(m3) & $\mathbf{1 0 3 . 8 4 0}$ & $\mathbf{9 2 . 2 8 6}$ & $\mathbf{8 8 . 9 0 4}$ & $\mathbf{8 7 . 3 4 3}$ \\
\hline
\end{tabular}

Table 4: Quantity of steel in column (trial 1)

\begin{tabular}{|l|l|l|l|l|}
\hline QUANTITY OF STEEL (FLOOR WISE) \\
\hline FLOOR NO. & CASE A & CASE B & CASE C & CASE D \\
\hline 1 & 2169.89 & 2053.460 & 1847.690 & 1706.140 \\
\hline 2 & 2649.42 & 2348.740 & 2242.250 & 2035.620 \\
\hline 3 & 2200.99 & 1821.400 & 1840.930 & 1914.840 \\
\hline 4 & 2181.70 & 1774.620 & 1752.490 & 1628.250 \\
\hline 5 & 1896.10 & 1587.010 & 1454.840 & 1535.240 \\
\hline 6 & 1641.19 & 1399.060 & 1188.940 & 1227.260 \\
\hline 7 & 1173.51 & 1079.690 & 1077.710 & 1078.950 \\
\hline 8 & 1173.51 & 1085.610 & 1079.690 & 1080.920 \\
\hline 9 & 1184.61 & 1094.730 & 1092.510 & 1091.540 \\
\hline 10 & 1217.90 & 1122.100 & 1114.460 & 1118.160 \\
\hline TOTAL $(\mathbf{k g})$ & $\mathbf{1 7 3 9 9 . 3 8}$ & $\mathbf{1 5 4 5 1 . 2 9 0}$ & $\mathbf{1 4 6 9 1 . 5 3 0}$ & $\mathbf{1 4 4 1 6 . 9 2 0}$ \\
\hline
\end{tabular}




\subsubsection{Footing}

Table 5: Quantity of concrete in footing (trial 1)

\begin{tabular}{|l|l|l|l|l|}
\hline \multicolumn{6}{|l|}{ QUANTITY OF CONCRETE (FLOOR WISE) } \\
\hline FLOOR NO. & CASE A & CASE B & CASE C & CASE D \\
\hline $\begin{array}{l}\text { FOOTING } \\
\text { LEVEL }\end{array}$ & 62.867 & 48.069 & 45.629 & 45.240 \\
\hline TOTAL(m3) & $\mathbf{6 2 . 8 6 7}$ & $\mathbf{4 8 . 0 6 9}$ & $\mathbf{4 5 . 6 2 9}$ & $\mathbf{4 5 . 2 4 0}$ \\
\hline
\end{tabular}

Table 6: Quantity of steel in footing (trial 1)

\begin{tabular}{|l|l|l|l|l|}
\hline \multicolumn{5}{|l|}{ QUANTITY OF steel (FLOOR WISE) } \\
\hline FLOOR NO. & CASE A & CASE B & CASE C & CASE D \\
\hline $\begin{array}{l}\text { FOOTING } \\
\text { LEVEL }\end{array}$ & 1677.47 & 1265.413 & 1203.913 & 1178.789 \\
\hline TOTAL(kg) & $\mathbf{1 6 7 7 . 4 7}$ & $\mathbf{1 2 6 5 . 4 1 3}$ & $\mathbf{1 2 0 3 . 9 1 3}$ & $\mathbf{1 1 7 8 . 7 8 9}$ \\
\hline
\end{tabular}

\subsubsection{Shear Wall}

Table 7: Quantity of concrete in shear wall (trial 1)

\begin{tabular}{|l|l|l|l|l|}
\hline \multicolumn{2}{|l|}{ QUANTITY OF CONCRETE (FLOOR WISE) } \\
\hline FLOOR NO. & CASE A & CASE B & CASE C & CASE D \\
\hline 1 & -- & 4.59 & 4.59 & 4.59 \\
\hline 2 & -- & 5.4 & 5.4 & 5.4 \\
\hline 3 & -- & 5.4 & 5.4 & 5.4 \\
\hline 4 & -- & 5.4 & 5.4 & 5.4 \\
\hline 5 & -- & 5.4 & 5.4 & 5.4 \\
\hline 6 & -- & 5.4 & 5.4 & 5.4 \\
\hline 7 & -- & 5.4 & 5.4 & 5.4 \\
\hline 8 & -- & 5.4 & 5.4 & 5.4 \\
\hline 9 & -- & 5.4 & 5.4 & 5.4 \\
\hline 10 & -- & 5.4 & 5.4 & 5.4 \\
\hline TOTAL(m3) & -- & $\mathbf{5 3 . 1 9}$ & $\mathbf{5 3 . 1 9}$ & $\mathbf{5 3 . 1 9}$ \\
\hline
\end{tabular}

Table 8: Quantity of steel in shear wall (trial 1)

\begin{tabular}{|c|c|c|c|c|}
\hline \multicolumn{5}{|c|}{ QUANTITY OF STEEL (FLOOR WISE) } \\
\hline FLOOR NO. & CASE A & CASE B & CASE C & CASE D \\
\hline 1 & -- & 663.33 & 993.52 & 812.44 \\
\hline 2 & -- & 513.11 & 770.00 & 682.3 \\
\hline 3 & -- & 171.78 & 413.56 & 342.44 \\
\hline 4 & -- & 171.78 & 171.78 & 171.78 \\
\hline 5 & -- & 171.78 & 171.78 & 203.78 \\
\hline 6 & -- & 171.78 & 171.78 & 203.78 \\
\hline 7 & -- & 171.78 & 171.78 & 203.78 \\
\hline 8 & -- & 171.78 & 171.78 & 203.78 \\
\hline 9 & -- & 171.78 & 171.78 & 171.78 \\
\hline 10 & -- & 235.78 & 171.78 & 171.78 \\
\hline TOTAL(kg) & -- & 2614.68 & 3379.54 & 3167.64 \\
\hline
\end{tabular}




\subsubsection{Trial:-2}

$>$ Live Load $=2 \mathrm{KN} / \mathrm{m} 2$

$>$ Preliminary Beam Size $=230 \times 300 \mathrm{~mm}$

\subsubsection{Beam}

Table 9: Quantity of concrete in beam (trial 2)

\begin{tabular}{|l|l|l|l|l|}
\hline \multicolumn{2}{|l|}{ QUANTITY OF CONCRETE (FLOOR WISE) } \\
\hline FLOOR NO. & CASE A & CASE B & CASE C & CASE D \\
\hline 1 & 17.388 & 17.595 & 19.665 & 16.870 \\
\hline 2 & 17.388 & 20.441 & 19.665 & 17.491 \\
\hline 3 & 17.388 & 17.595 & 17.181 & 16.767 \\
\hline 4 & 17.388 & 17.595 & 17.181 & 16.870 \\
\hline 5 & 17.388 & 17.595 & 17.181 & 16.767 \\
\hline 6 & 17.388 & 17.565 & 17.181 & 16.767 \\
\hline 7 & 17.388 & 16.560 & 16.560 & 16.663 \\
\hline 8 & 17.388 & 16.560 & 16.560 & 16.360 \\
\hline 9 & 17.388 & 16.560 & 16.560 & 16.560 \\
\hline 10 & 17.388 & 16.560 & 16.560 & 16.560 \\
\hline TOTAL(m3) & 173.88 & $\mathbf{1 5 7 . 0 6 1}$ & $\mathbf{1 7 4 . 2 9 4}$ & $\mathbf{1 6 7 . 6 7 5}$ \\
\hline
\end{tabular}

Table 10: Quantity of steel in beam (trial 2)

\begin{tabular}{|l|l|l|l|l|}
\hline \multicolumn{4}{|l|}{ QUANTITY OF STEEL (FLOOR WISE) } \\
\hline FLOOR NO. & CASE A & CASE B & CASE C & CASE D \\
\hline 1 & 3717.462 & 3123.434 & 3032.986 & 3509.906 \\
\hline 2 & 4024.229 & 3672.450 & 3032.986 & 4547.092 \\
\hline 3 & 2191.105 & 2354.751 & 2763.507 & 2824.756 \\
\hline 4 & 2135.395 & 2358.587 & 2868.484 & 2915.473 \\
\hline 5 & 2090.489 & 2369.129 & 2877.104 & 2849.714 \\
\hline 6 & 2011.030 & 2343.593 & 2808.405 & 2748.934 \\
\hline 7 & 1898.195 & 2751.080 & 2889.675 & 2625.218 \\
\hline 8 & 1848.690 & 2317.591 & 2782.623 & 2501.200 \\
\hline 9 & 1758.512 & 2225.688 & 2646.002 & 2372.185 \\
\hline 10 & 1572.402 & 1796.802 & 2388.273 & 1996.810 \\
\hline TOTAL(kg) & $\mathbf{2 3 1 6 8 . 4 4 7}$ & $\mathbf{2 5 0 1 7 . 1 0 5}$ & $\mathbf{2 8 0 9 0 . 0 4 5}$ & $\mathbf{2 8 8 9 1 . 2 7 8}$ \\
\hline
\end{tabular}

\subsubsection{Column}

Table 11: Quantity of concrete in column (trial 2)

\begin{tabular}{|l|l|l|l|l|}
\hline QUANTITY OF CONCRETE (FLOOR WISE) \\
\hline FLOOR NO. & CASE A & CASE B & CASE C & CASE D \\
\hline 1 & 13.296 & 11.874 & 12.185 & 11.887 \\
\hline 2 & 16.008 & 15.554 & 15.554 & 15.341 \\
\hline 3 & 14.910 & 13.970 & 12.140 & 12.963 \\
\hline 4 & 13.446 & 13.238 & 11.041 & 10.858 \\
\hline 5 & 11.525 & 11.316 & 10.584 & 10.767 \\
\hline 6 & 11.525 & 11.316 & 09.972 & 10.690 \\
\hline 7 & 07.776 & 10.016 & 08.748 & 08.671 \\
\hline 8 & 07.776 & 09.403 & 08.059 & 08.059 \\
\hline 9 & 07.776 & 09.403 & 08.059 & 07.600 \\
\hline 10 & 08.082 & 09.403 & 08.059 & 08.136 \\
\hline
\end{tabular}


\begin{tabular}{|l|l|}
\hline TOTAL(m3) & $\mathbf{1 1 2 . 1 2 2}$ \\
\hline
\end{tabular}

115.493

104.402

104.974

Table 12: Quantity of steel in column (trial 2)

\begin{tabular}{|l|l|l|l|l|}
\hline \multicolumn{6}{|l|}{ QUANTITY OF STEEL (FLOOR WISE) } \\
\hline FLOOR NO. & CASE A & CASE B & CASE C & CASE D \\
\hline 1 & 2482.22 & 3976.700 & 2002.500 & 1827.04 \\
\hline 2 & 3319.08 & 2965.530 & 2873.540 & 1868.10 \\
\hline 3 & 2347.13 & 2094.710 & 2068.410 & 1997.40 \\
\hline 4 & 2139.91 & 2059.060 & 1989.230 & 2119.35 \\
\hline 5 & 1992.30 & 1960.880 & 1888.160 & 1807.48 \\
\hline 6 & 1881.07 & 1849.660 & 1809.730 & 1731.25 \\
\hline 7 & 1778.99 & 1814.280 & 1776.420 & 1987.83 \\
\hline 8 & 1173.51 & 1587.580 & 1448.730 & 1349.86 \\
\hline 9 & 1222.95 & 1488.660 & 1250.990 & 1337.38 \\
\hline 10 & 1231.47 & 1636.960 & 1646.460 & 1340.35 \\
\hline TOTAL(kg) & $\mathbf{1 9 5 6 8 . 6 3}$ & $\mathbf{1 9 6 6 9 . 9 7 0}$ & $\mathbf{1 8 7 0 7 . 2 3 0}$ & $\mathbf{1 8 0 8 4 . 0 3}$ \\
\hline
\end{tabular}

\subsubsection{Footing}

Table 13: Quantity of concrete in footing (trial 2)

\begin{tabular}{|l|l|l|l|l|}
\hline \multicolumn{4}{|l|}{ QUANTITY OF CONCRETE (FLOOR WISE) } \\
\hline FLOOR NO. & CASE A & CASE B & CASE C & CASE D \\
\hline $\begin{array}{l}\text { FOOTING } \\
\text { LEVEL }\end{array}$ & 60.341 & 46.945 & 47.211 & 45.801 \\
\hline TOTAL(m3) & $\mathbf{6 0 . 3 4 1}$ & $\mathbf{4 6 . 9 4 5}$ & $\mathbf{4 7 . 2 1 1}$ & $\mathbf{4 5 . 8 0 1}$ \\
\hline
\end{tabular}

Table 14: Quantity of steel in footing (trial 2)

\begin{tabular}{|l|l|l|l|l|}
\hline QUANTITY OF steel (FLOOR WISE) \\
\hline FLOOR NO. & CASE A & CASE B & CASE C & CASE D \\
\hline $\begin{array}{l}\text { FOOTING } \\
\text { LEVEL }\end{array}$ & 1680.016 & 1287.765 & 1326.009 & 1300.167 \\
\hline TOTAL(kg) & $\mathbf{1 6 8 0 . 0 1 6}$ & $\mathbf{1 2 8 7 . 7 6 5}$ & $\mathbf{1 3 2 6 . 0 0 9}$ & $\mathbf{1 3 0 0 . 1 6 7}$ \\
\hline
\end{tabular}

\subsubsection{Shear Wall}

Table 15: Quantity of concrete in shear wall (trial 2)

\begin{tabular}{|l|l|l|l|l|}
\hline \multicolumn{2}{|l|}{ QUANTITY OF CONCRETE (FLOOR WISE) } \\
\hline FLOOR NO. & CASE A & CASE B & CASE C & CASE D \\
\hline 1 & -- & 4.590 & 4.590 & 4.590 \\
\hline 2 & -- & 5.400 & 5.400 & 5.400 \\
\hline 3 & -- & 5.400 & 5.400 & 5.400 \\
\hline 4 & -- & 5.400 & 5.400 & 5.400 \\
\hline 5 & -- & 5.400 & 5.400 & 5.400 \\
\hline 6 & -- & 5.400 & 5.400 & 5.400 \\
\hline 7 & -- & 5.400 & 5.400 & 5.400 \\
\hline 8 & -- & 5.400 & 5.400 & 5.400 \\
\hline 9 & -- & 5.400 & 5.400 & 5.400 \\
\hline 10 & -- & 5.400 & 5.400 & 5.400 \\
\hline TOTAL(m3) & -- & $\mathbf{5 3 . 1 9}$ & $\mathbf{5 3 . 1 9}$ & $\mathbf{5 3 . 1 9}$ \\
\hline
\end{tabular}


Table 16: Quantity of steel in shear wall (trial 2)

\begin{tabular}{|c|c|c|c|c|}
\hline \multicolumn{5}{|c|}{ QUANTITY OF STEEL (FLOOR WISE) } \\
\hline FLOOR NO. & CASE A & CASE B & CASE C & CASE D \\
\hline 1 & -- & 993.49 & 993.52 & 1294.99 \\
\hline 2 & -- & 770.00 & 769.60 & 547.18 \\
\hline 3 & -- & 314.00 & 413.56 & 242.89 \\
\hline 4 & -- & 171.78 & 171.78 & 171.78 \\
\hline 5 & -- & 171.78 & 171.78 & 203.78 \\
\hline 6 & -- & 171.78 & 171.78 & 203.78 \\
\hline 7 & -- & 171.78 & 171.78 & 242.89 \\
\hline 8 & -- & 171.78 & 171.78 & 203.78 \\
\hline 9 & -- & 171.78 & 171.78 & 203.78 \\
\hline 10 & -- & 171.78 & 171.78 & 171.79 \\
\hline TOTAL(kg) & -- & 3279.97 & 3379.64 & 3486.74 \\
\hline
\end{tabular}

\subsubsection{Trial:-3}

$>$ Live Load $=3 \mathrm{KN} / \mathrm{m} 2$

$>$ Preliminary Beam Size $=230 \times 300 \mathrm{~mm}$

\subsubsection{Beam}

Table 17: Quantity of concrete in beam (trial 3)

\begin{tabular}{|l|l|l|l|l|}
\hline \multicolumn{2}{|l|}{ QUANTITY OF CONCRETE (FLOOR WISE) } \\
\hline FLOOR NO. & CASE A & CASE B & CASE C & CASE D \\
\hline 1 & 17.388 & 16.560 & 16.560 & 16.560 \\
\hline 2 & 17.388 & 16.870 & 17.181 & 16.663 \\
\hline 3 & 17.388 & 16.560 & 16.560 & 16.560 \\
\hline 4 & 17.388 & 16.560 & 16.560 & 16.560 \\
\hline 5 & 17.388 & 16.560 & 16.560 & 16.560 \\
\hline 6 & 17.388 & 16.560 & 16.560 & 16.560 \\
\hline 7 & 17.388 & 16.560 & 16.560 & 16.560 \\
\hline 8 & 17.388 & 16.560 & 16.560 & 16.560 \\
\hline 9 & 17.388 & 16.560 & 16.560 & 16.560 \\
\hline 10 & 17.388 & 16.560 & 16.560 & 16.560 \\
\hline TOTAL(m3) & $\mathbf{1 7 3 . 8 8 0}$ & $\mathbf{1 6 5 . 9 1}$ & $\mathbf{1 6 6 . 2 2 1}$ & $\mathbf{1 6 5 . 7 0 3}$ \\
\hline
\end{tabular}

Table 18: Quantity of steel in beam (trial 3)

\begin{tabular}{|l|l|l|l|l|}
\hline \multicolumn{2}{|l|}{ QUANTITY OF STEEL (FLOOR WISE) } \\
\hline FLOOR NO. & CASE A & CASE B & CASE C & CASE D \\
\hline 1 & 3409.881 & 2016.202 & 1973.808 & 2031.858 \\
\hline 2 & 3715.175 & 2570.800 & 2647.202 & 2706.889 \\
\hline 3 & 2122.567 & 1944.847 & 2218.602 & 2186.091 \\
\hline 4 & 2081.692 & 2055.334 & 2345.550 & 2250.877 \\
\hline 5 & 2057.334 & 2096.149 & 2418.503 & 2291.698 \\
\hline 6 & 1979.012 & 2121.062 & 2448.692 & 2308.634 \\
\hline 7 & 1930.206 & 2119.877 & 2438.504 & 2331.549 \\
\hline 8 & 1893.578 & 2108.370 & 2445.335 & 2293.049 \\
\hline 9 & 1823.225 & 2067.440 & 2462.595 & 2292.016 \\
\hline 10 & 1573.728 & 1776.462 & 2346.312 & 2038.324 \\
\hline TOTAL(kg) & 22586.398 & $\mathbf{2 0 8 7 6 . 5 4 3}$ & $\mathbf{2 3 7 4 5 . 1 0 3}$ & $\mathbf{2 2 7 3 0 . 9 8 5}$ \\
\hline
\end{tabular}




\subsubsection{Column}

Table 19: Quantity of concrete in column (trial 3)

\begin{tabular}{|l|l|l|l|l|}
\hline \multicolumn{2}{|l|}{ QUANTITY OF CONCRETE (FLOOR WISE) } \\
\hline FLOOR NO. & CASE A & CASE B & CASE C & CASE D \\
\hline 1 & 13.296 & 11.354 & 10.319 & 11.627 \\
\hline 2 & 15.642 & 13.970 & 12.872 & 13.572 \\
\hline 3 & 13.812 & 11.894 & 11.102 & 10.567 \\
\hline 4 & 11.219 & 9.972 & 9.757 & 9.895 \\
\hline 5 & 10.607 & 9.360 & 8.442 & 8.442 \\
\hline 6 & 7.465 & 7.830 & 7.524 & 7.141 \\
\hline 7 & 7.776 & 7.141 & 7.141 & 7.141 \\
\hline 8 & 7.776 & 7.141 & 7.141 & 7.141 \\
\hline 9 & 7.776 & 7.141 & 7.141 & 7.141 \\
\hline 10 & 7.776 & 7.141 & 7.141 & 7.141 \\
\hline TOTAL(m3) & $\mathbf{1 0 4 . 1 4 5}$ & $\mathbf{9 2 . 9 4 4}$ & $\mathbf{8 8 . 3 9 8}$ & $\mathbf{8 9 . 8 0 8}$ \\
\hline
\end{tabular}

Table 20: Quantity of steel in column (trial 3)

\begin{tabular}{|l|l|l|l|l|}
\hline \multicolumn{4}{|l|}{ QUANTITY OF STEEL (FLOOR WISE) } \\
\hline FLOOR NO. & CASE A & CASE B & CASE C & CASE D \\
\hline 1 & 2323.87 & 2049.77 & 1951.030 & 1627.15 \\
\hline 2 & 2591.19 & 2247.33 & 2231.62 & 1866.91 \\
\hline 3 & 2312.21 & 1968.04 & 1906.04 & 2074.78 \\
\hline 4 & 2148.64 & 1829.47 & 1804.31 & 1684.06 \\
\hline 5 & 1806.66 & 1595.34 & 1520.93 & 1558.01 \\
\hline 6 & 1649.51 & 1405.41 & 1298.22 & 1287.81 \\
\hline 7 & 1284.74 & 1188.94 & 1139.51 & 1077.71 \\
\hline 8 & 1173.51 & 1077.71 & 1077.71 & 1077.71 \\
\hline 9 & 1173.51 & 1077.71 & 1077.71 & 1077.71 \\
\hline 10 & 1173.51 & 1077.71 & 1077.71 & 1077.71 \\
\hline TOTAL(kg) & $\mathbf{1 7 6 7 3 . 3 5}$ & $\mathbf{1 5 5 1 7 . 4 3}$ & $\mathbf{1 5 0 8 4 . 7 9}$ & $\mathbf{1 4 4 0 9 . 5 6}$ \\
\hline
\end{tabular}

\subsubsection{Footing}

Table 21: Quantity of concrete in footing (trial 3)

\begin{tabular}{|l|l|l|l|l|}
\hline \multicolumn{2}{|l|}{ QUANTITY OF CONCRETE (FLOOR WISE) } & CASE C & CASE D \\
\hline FLOOR NO. & CASE A & CASE B & 50.479 & 50.331 \\
\hline $\begin{array}{l}\text { FOOTING } \\
\text { LEVEL }\end{array}$ & 66.548 & 56.888 & & \\
\hline TOTAL $(\mathbf{m 3})$ & $\mathbf{6 6 . 5 4 8}$ & $\mathbf{5 6 . 8 8 8}$ & $\mathbf{5 0 . 4 7 9}$ & $\mathbf{5 0 . 3 3 1}$ \\
\hline
\end{tabular}

Table 22: Quantity of steel in footing (trial 3)

\begin{tabular}{|l|l|l|l|l|}
\hline \multicolumn{4}{|l|}{ QUANTITY OF STEEL (FLOOR WISE) } \\
\hline FLOOR NO. & CASE A & CASE B & CASE C & CASE D \\
\hline $\begin{array}{l}\text { FOOTING } \\
\text { LEVEL }\end{array}$ & 1802.823 & 1527.548 & 1347.562 & 1331.036 \\
\hline TOTAL(kg) & $\mathbf{1 8 0 2 . 8 2 3}$ & $\mathbf{1 5 2 7 . 5 4 8}$ & $\mathbf{1 3 4 7 . 5 6 2}$ & $\mathbf{1 3 3 1 . 0 3 6}$ \\
\hline
\end{tabular}




\subsubsection{Shear Wall}

Table 23: Quantity of concrete in shear wall (trial 3)

\begin{tabular}{|l|l|l|l|l|}
\hline \multicolumn{2}{|l|}{ QUANTITY OF CONCRETE (FLOOR WISE) } \\
\hline FLOOR NO. & CASE A & CASE B & CASE C & CASE D \\
\hline 1 & -- & 4.59 & 4.590 & 4.590 \\
\hline 2 & -- & 5.40 & 5.400 & 5.400 \\
\hline 3 & -- & 5.40 & 5.400 & 5.400 \\
\hline 4 & -- & 5.40 & 5.400 & 5.400 \\
\hline 5 & -- & 5.40 & 5.400 & 5.400 \\
\hline 6 & -- & 5.40 & 5.400 & 5.400 \\
\hline 7 & -- & 5.40 & 5.400 & 5.400 \\
\hline 8 & -- & 5.40 & 5.400 & 5.400 \\
\hline 9 & -- & 5.40 & 5.400 & 5.400 \\
\hline 10 & -- & 5.40 & 5.400 & 5.400 \\
\hline TOTAL(m3) & -- & $\mathbf{5 3 . 1 9}$ & $\mathbf{5 3 . 1 9}$ & $\mathbf{5 3 . 1 9}$ \\
\hline
\end{tabular}

Table 24: Quantity of steel in shear wall (trial 3)

\begin{tabular}{|c|c|c|c|c|}
\hline \multicolumn{5}{|c|}{ QUANTITY OF STEEL (FLOOR WISE) } \\
\hline FLOOR NO. & CASE A & CASE B & CASE C & CASE D \\
\hline 1 & -- & 653.52 & 993.52 & 812.44 \\
\hline 2 & -- & 514.00 & 770.00 & 682.30 \\
\hline 3 & -- & 171.78 & 413.56 & 342.44 \\
\hline 4 & -- & 171.78 & 171.78 & 171.78 \\
\hline 5 & -- & 171.78 & 171.78 & 203.78 \\
\hline 6 & -- & 171.78 & 171.78 & 203.78 \\
\hline 7 & -- & 171.78 & 171.78 & 203.78 \\
\hline 8 & -- & 171.78 & 171.78 & 203.78 \\
\hline 9 & -- & 171.78 & 171.78 & 171.78 \\
\hline 10 & -- & 235.78 & 171.78 & 171.78 \\
\hline TOTAL(kg) & -- & 2605.75 & 3379.53 & 3167.64 \\
\hline
\end{tabular}

\subsection{Discussion}

\subsubsection{Trial: - 1}

Table 25:- Quantity of concrete \& steel in beam (trial 1)

\begin{tabular}{|l|l|l|}
\hline \multicolumn{2}{|l|}{ QUANTITY OF CONCRETE \& STEEL IN BEAM (CASE WISE) } \\
\hline CASE & QUANTITY OF CONCRETE (m3) & QUANTITY OF STEEL (Kg) \\
\hline A & 260.82 & 21425.693 \\
\hline B & 249.917 & 24188.575 \\
\hline C & 249.642 & 28502.922 \\
\hline D & 250.297 & 27874.944 \\
\hline
\end{tabular}

Table 26:- Quantity of concrete \& steel in column (trial 1)

\begin{tabular}{|l|l|l|}
\hline \multicolumn{3}{|l|}{ QUANTITY OF CONCRETE \& STEEL IN COLUMN (CASE WISE) } \\
\hline CASE & QUANTITY OF CONCRETE (m3) & QUANTITY OF STEEL (Kg) \\
\hline A & 103.840 & 17399.38 \\
\hline
\end{tabular}




\begin{tabular}{|l|l|l|}
\hline B & 92.286 & 15451.290 \\
\hline C & 88.904 & 14691.530 \\
\hline D & 87.343 & 14416.920 \\
\hline
\end{tabular}

Table 27:- Quantity of concrete \& steel in footing (trial 1) QUANTITY OF CONCRETE \& STEEL IN FOOTING (CASE WISE)

\begin{tabular}{|l|l|l|}
\hline CASE & QUANTITY OF CONCRETE (m3) & QUANTITY OF STEEL (Kg) \\
\hline A & 62.867 & 1677.47 \\
\hline B & 48.069 & 1265.413 \\
\hline C & 45.629 & 1203.913 \\
\hline D & 45.240 & 1178.789 \\
\hline
\end{tabular}

Table 28:- Quantity of concrete \& steel in shear wall (trial 1)

\begin{tabular}{|l|l|l|}
\hline \multicolumn{2}{|l|}{ QUANTITY OF CONCRETE \& STEEL IN SHEAR WALL (CASE WISE) } \\
\hline CASE & QUANTITY OF CONCRETE (m3) & QUANTITY OF STEEL (Kg) \\
\hline A & --- & --- \\
\hline B & 53.19 & 2614.68 \\
\hline C & 53.19 & 3379.54 \\
\hline D & 53.19 & 3167.64 \\
\hline
\end{tabular}

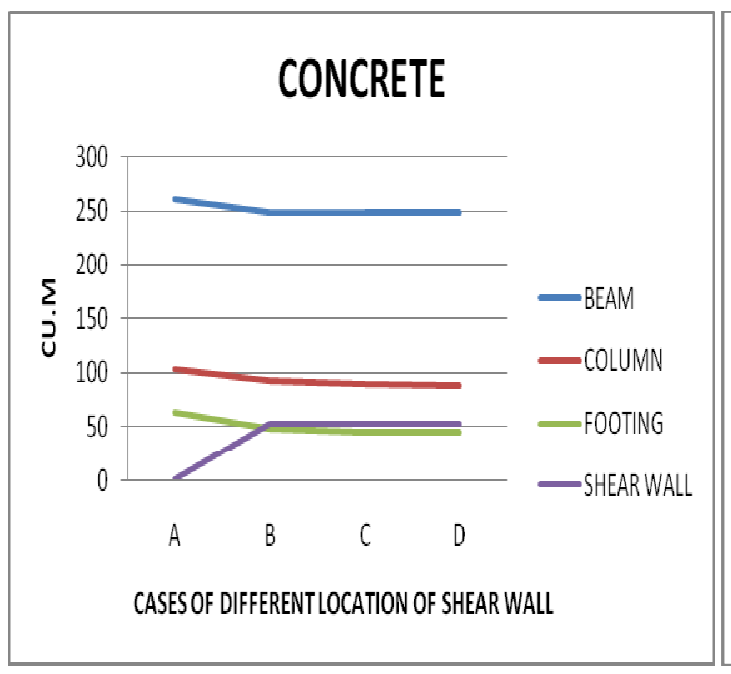

Fig 5 :- Comparision of quantity of concrete of each component case wise (trial 1)

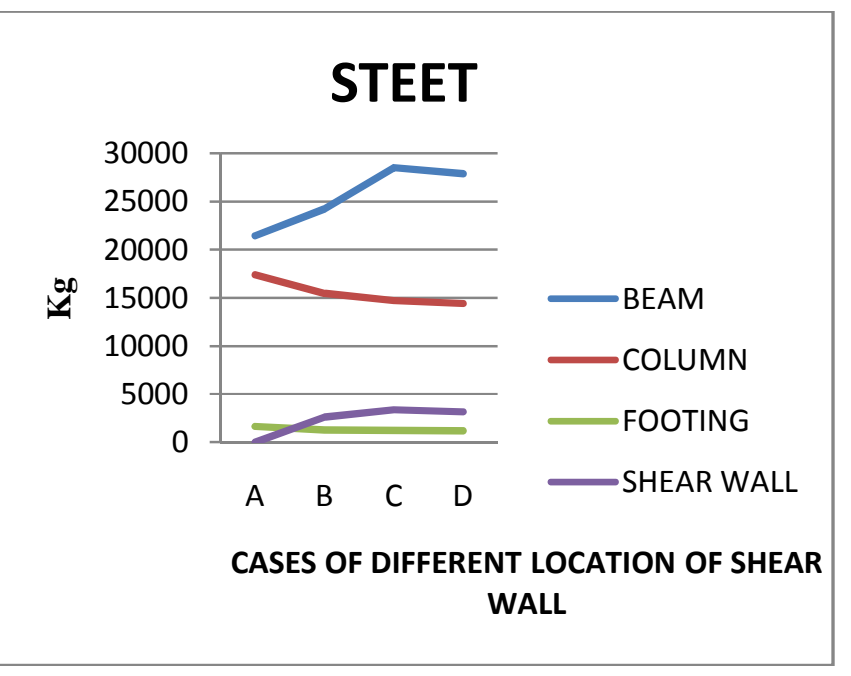

Fig 6:- Comparision of quantity of steel of of each component case wise (trial 1)

Table 29:- Total quantity of concrete \& steel (trial 1)

\begin{tabular}{|l|l|l|}
\hline \multicolumn{3}{|c|}{ TOTAL QUANTITY OF CONCRETE \& STEEL (CASE WISE) } \\
\hline CASE & QUANTITY OF CONCRETE (m3) & QUANTITY OF STEEL (Kg) \\
\hline A & 427.527 & 40502.543 \\
\hline B & 443.462 & 43519.958 \\
\hline
\end{tabular}




\begin{tabular}{|l|l|l|}
\hline C & 437.365 & 47777.905 \\
\hline D & 436.07 & 46638.293 \\
\hline
\end{tabular}

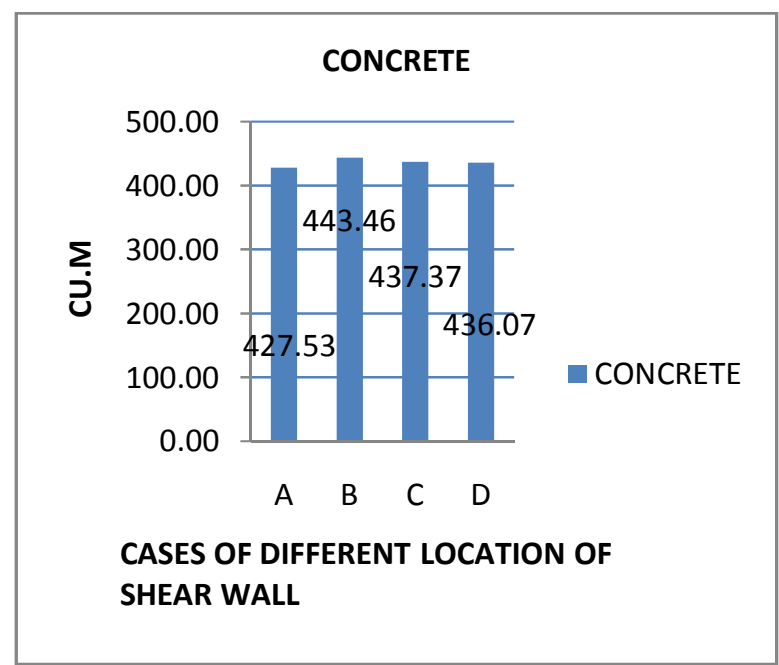

Fig 7:- Comparision of total quantity of concrete case wise (trial 1)

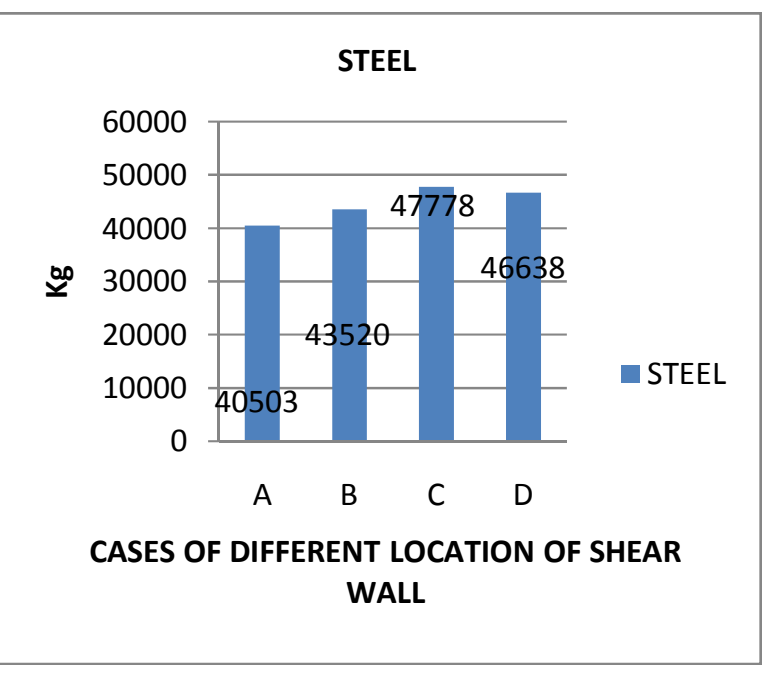

Fig 8:- Comparision of total quantity of steel case wise (trial 1)

\subsubsection{Trial: - 2}

Table 30:- Quantity of concrete \& steel in beam (trial 2)

\begin{tabular}{|l|l|l|}
\hline \multicolumn{2}{|l|}{ QUANTITY OF CONCRETE \& STEEL IN BEAM (CASE WISE) } \\
\hline CASE & QUANTITY OF CONCRETE (m3) & QUANTITY OF STEEL (Kg) \\
\hline A & 173.88 & 23168.447 \\
\hline B & 157.061 & 25017.105 \\
\hline C & 174.294 & 28090.045 \\
\hline D & 167.675 & 28891.278 \\
\hline
\end{tabular}

Table 31:- Quantity of concrete \& steel in column (trial 2)

\begin{tabular}{|l|l|l|}
\hline \multicolumn{2}{|l|}{ QUANTITY OF CONCRETE \& STEEL IN COLUMN (CASE WISE) } \\
\hline CASE & QUANTITY OF CONCRETE (m3) & QUANTITY OF STEEL (Kg) \\
\hline A & 112.122 & 19568.630 \\
\hline B & 115.493 & 19669.097 \\
\hline C & 104.402 & 18707.230 \\
\hline D & 104.974 & 18084.030 \\
\hline
\end{tabular}

Table 32:- Quantity of concrete \& steel in footing (trial 2)

\begin{tabular}{|l|l|l|}
\hline \multicolumn{3}{|l|}{ QUANTITY OF CONCRETE \& STEEL IN FOOTING (CASE WISE) } \\
\hline CASE & QUANTITY OF CONCRETE (m3) & QUANTITY OF STEEL (Kg) \\
\hline A & 60.341 & 1680.016 \\
\hline B & 46.945 & 1287.765 \\
\hline
\end{tabular}




\begin{tabular}{|l|l|l|}
\hline C & 47.211 & 1326.009 \\
\hline $\mathbf{D}$ & 45.801 & 1300.167 \\
\hline
\end{tabular}

Table 33:- Quantity of concrete \& steel in shear wall (trial 2)

\begin{tabular}{|l|l|l|}
\hline \multicolumn{2}{|l|}{ QUANTITY OF CONCRETE \& STEEL IN SHEAR WALL (CASE WISE) } \\
\hline CASE & QUANTITY OF CONCRETE (m3) & QUANTITY OF STEEL (Kg) \\
\hline A & --- & --- \\
\hline B & 53.19 & 3279.97 \\
\hline C & 53.19 & 3378.64 \\
\hline D & 53.19 & 3166.74 \\
\hline
\end{tabular}

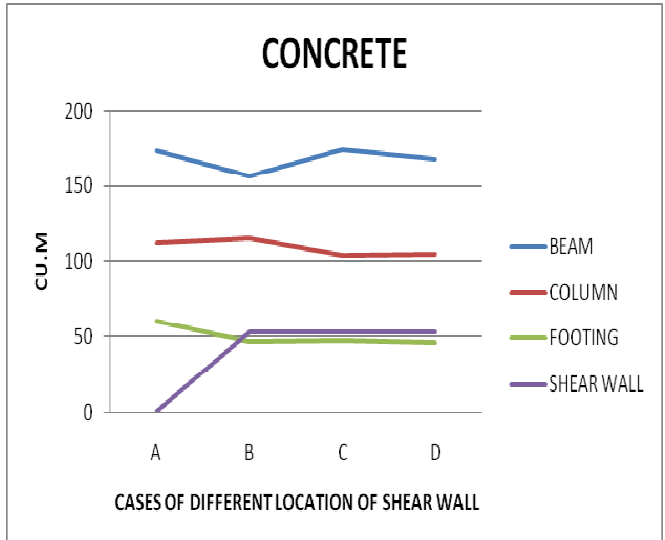

Fig 9:- Comparision of quantity of concrete of each component case wise (trial 2)

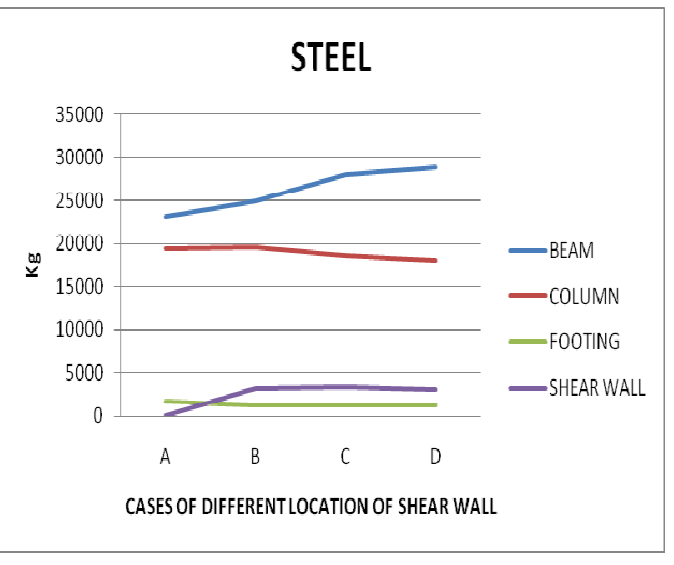

Fig 10:- Comparision of quantity of steel of each component case wise (trial 2)

Table 34:- Total quantity of concrete \& steel (trial 2)

\begin{tabular}{|l|l|l|}
\hline \multicolumn{3}{|l|}{ TOTAL QUANTITY OF CONCRETE \& STEEL (CASE WISE) } \\
\hline CASE & QUANTITY OF CONCRETE (m3) & QUANTITY OF STEEL (Kg) \\
\hline A & 346.343 & 44417.093 \\
\hline B & 372.689 & 49253.937 \\
\hline C & 379.097 & 51502.924 \\
\hline D & 371.64 & 51442.215 \\
\hline
\end{tabular}




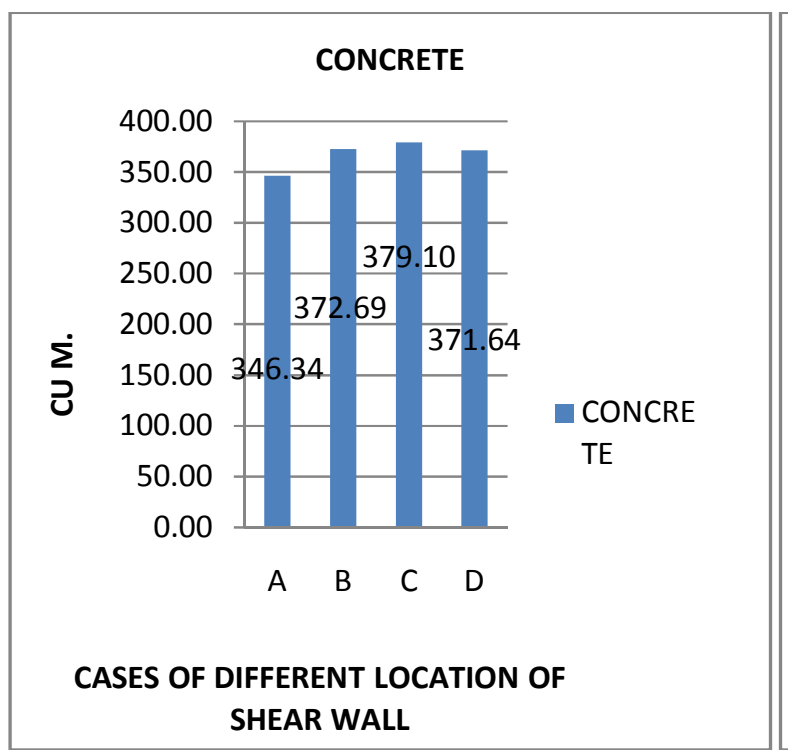

Fig 11:- Comparision of total quantity of concrete case wise (trial 2)

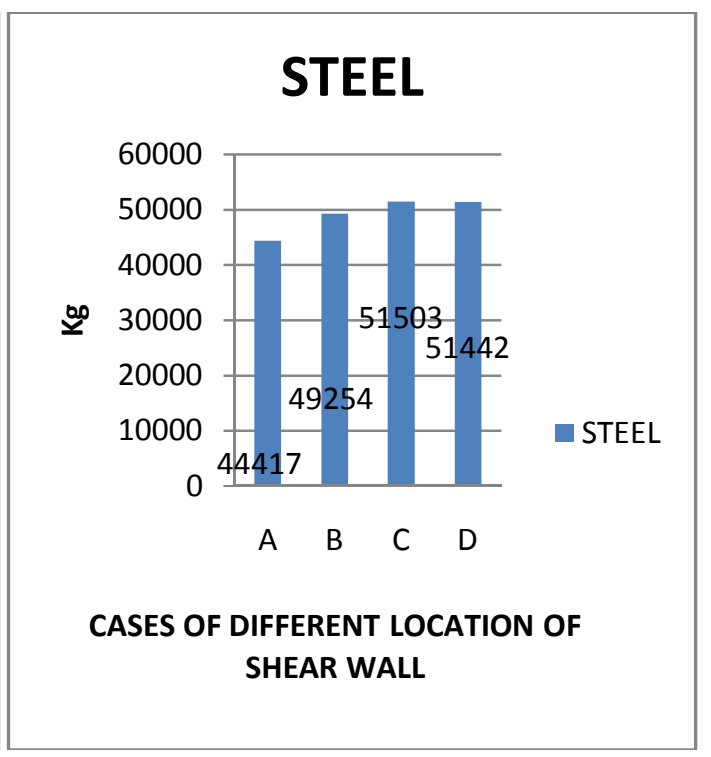

Fig 12:- Comparision of total quantity of concrete case wise (trial 2)

\subsubsection{Trial: -3}

Table 35:- Quantity of concrete \& steel in beam (trial 3)

\begin{tabular}{|l|l|l|}
\hline \multicolumn{2}{|l|}{ QUANTITY OF CONCRETE \& STEEL IN BEAM (CASE WISE) } \\
\hline CASE & QUANTITY OF CONCRETE (m3) & QUANTITY OF STEEL (Kg) \\
\hline A & 173.880 & 22586.398 \\
\hline B & 165.710 & 20876.543 \\
\hline C & 166.221 & 23745.103 \\
\hline D & 165.703 & 22730.985 \\
\hline
\end{tabular}

Table 36:- Quantity of concrete \& steel in column (trial 3)

\begin{tabular}{|l|l|l|}
\hline \multicolumn{3}{|l|}{ QUANTITY OF CONCRETE \& STEEL IN COLUMN (CASE WISE) } \\
\hline CASE & QUANTITY OF CONCRETE (m3) & QUANTITY OF STEEL (Kg) \\
\hline A & 104.145 & 17637.35 \\
\hline B & 92.944 & 15517.13 \\
\hline C & 88.398 & 15084.79 \\
\hline D & 89.808 & 14409.56 \\
\hline
\end{tabular}

Table 37:- Quantity of concrete \& steel in footing (trial 3)

\begin{tabular}{|l|l|l|}
\hline \multicolumn{2}{|l|}{ QUANTITY OF CONCRETE \& STEEL IN FOOTING (CASE WISE) } \\
\hline CASE & QUANTITY OF CONCRETE (m3) & QUANTITY OF STEEL (Kg) \\
\hline A & 66.548 & 1802.823 \\
\hline B & 56.888 & 1527.548 \\
\hline C & 50.479 & 1347.562 \\
\hline
\end{tabular}




\begin{tabular}{|l|l|l|}
\hline D & 50.331 & 1331.036 \\
\hline
\end{tabular}

Table 38:- Quantity of concrete \& steel in shear wall (trial 3)

\begin{tabular}{|l|l|l|}
\hline \multicolumn{2}{|l|}{ QUANTITY OF CONCRETE \& STEEL IN SHEAR WALL (CASE WISE) } \\
\hline CASE & QUANTITY OF CONCRETE (m3) & QUANTITY OF STEEL (Kg) \\
\hline A & --- & --- \\
\hline B & 53.19 & 2605.75 \\
\hline C & 53.19 & 3379.53 \\
\hline D & 53.19 & 3167.64 \\
\hline
\end{tabular}

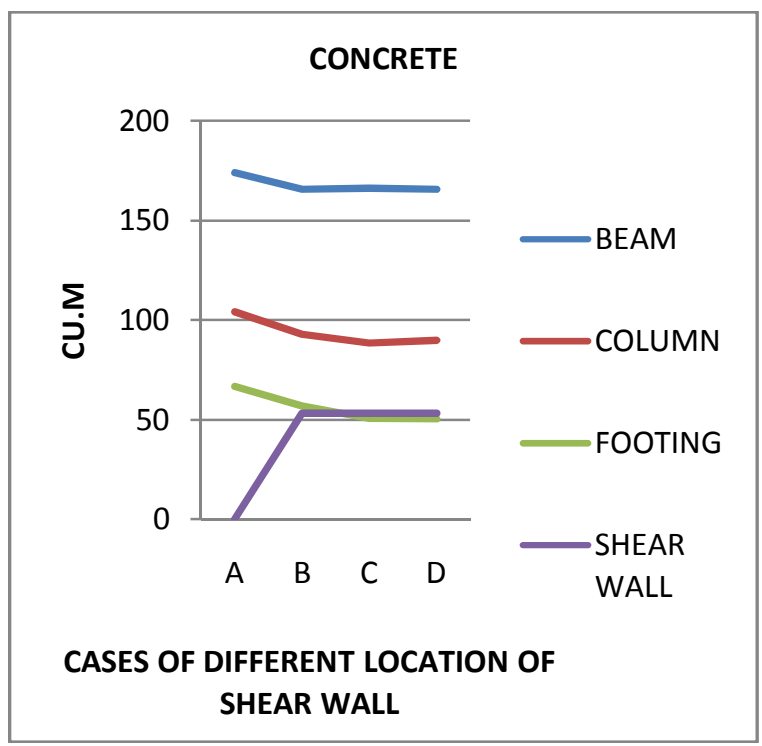

Fig 13:- Comparision of quantity of concrete of each component case wise (trial 3)

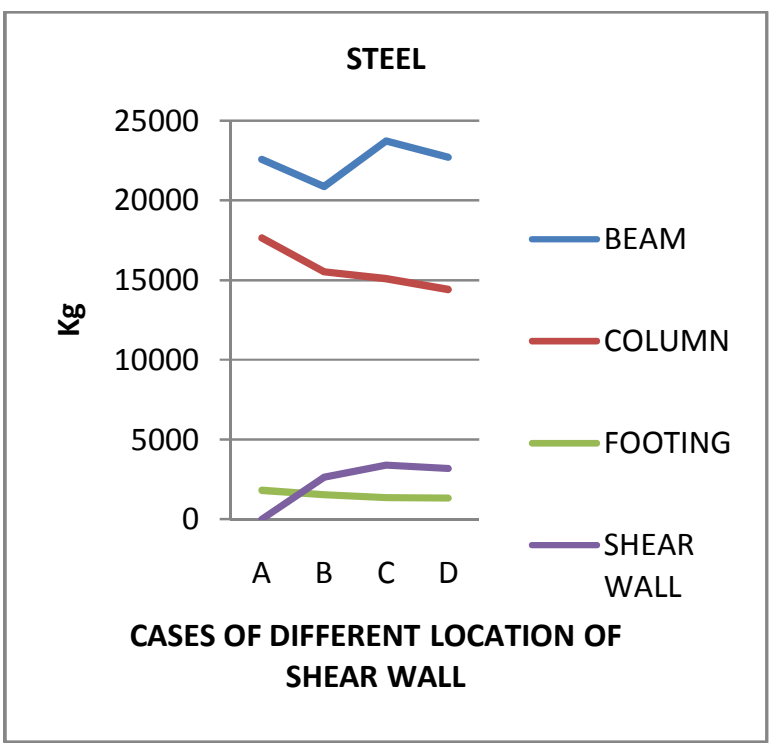

Fig 14:- Comparision of quantity of steel of each component case wise (trial 3)

Table 39:- Total quantity of concrete \& steel (trial 3)

\begin{tabular}{|l|l|l|}
\hline \multicolumn{3}{|l|}{ TOTAL QUANTITY OF CONCRETE \& STEEL (CASE WISE) } \\
\hline CASE & QUANTITY OF CONCRETE (m3) & QUANTITY OF STEEL (Kg) \\
\hline A & 344.573 & 42026.571 \\
\hline B & 368.732 & 40526.971 \\
\hline C & 358.288 & 43556.985 \\
\hline D & 359.032 & 41639.221 \\
\hline
\end{tabular}




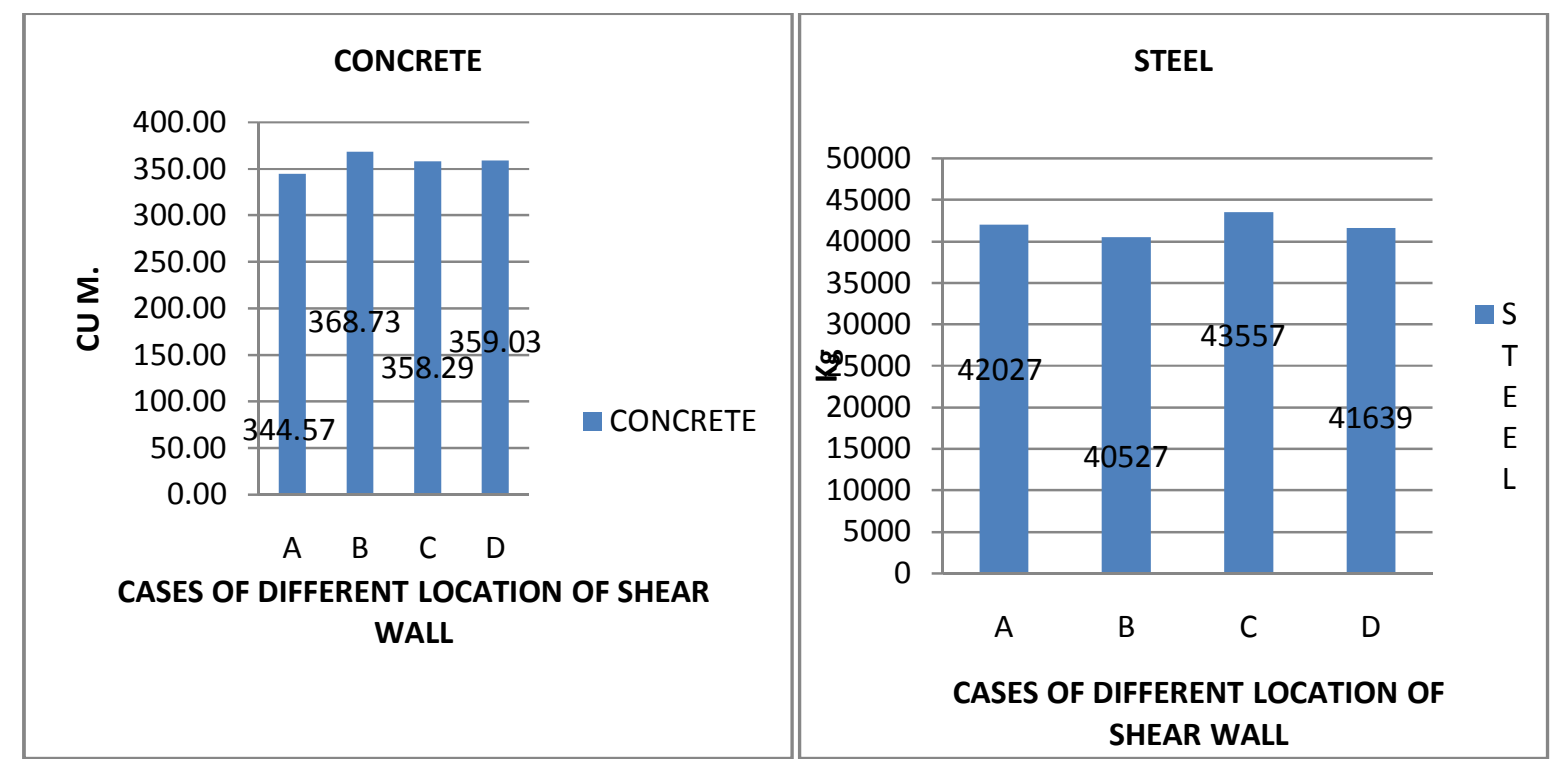

Fig 15:- Comparision of total quantity of concrete case wise (trial 2)

Fig 16:- Comparision of total quantity of concrete case wise (trial 2)

\section{REFERENCES}

[1]. Dr. Memari Ali, (2010), office building G, Eastern United States. Technical report, vol-3.

[2]. M.Ashraf, Siddiqi Z.A. \& Javed M.A., (2008), Configuration of a multistorey building subjected to lateral forces, vol-9, page no:-525-537.

[3]. Kaltakci M.Y., Arslan M.H.,Yavuz G., (2010), Effect of internal \& external shear wall location on strengthening weak RC frames, vol-17, page no:- 312-323.

[4]. Onkar V. Sapate, Dr.A.M.Pande/International journal of Engineering Research and Applications. Vol. 1, Issue 4. Pp.1515-152

[5]. IS: 456-code of practice for plain and reinforced concrete [6]. IS: 875(part 1-5)- code of practice for structural safety of building loading standards

[7]. IS 1893(Part-1):2002, Criteria for earthquake resistant design of structures.

[8]. IS 13920:1993,Ductile detailing of reinforced concrete structure subjected to seismic forces-code of practice.

[9]. SP: 16-design aids for reinforced concrete

[10]. Dr. Jain k., Explanatory example on indian seismic code IS 1893 (Part-1).

[11]. Dr. Shah H.J. \& Dr. Jain Sudhir k., Design example of a six storey building.

[12]. www.World-housing.net/wp-content/uploads/..../type-

RC-Wall.pdf

[13]. www.nibs.org/../Topic11-...

[14]. www.iitk.ac.in/nicee/EQTips/EQTip23.pdf

[15]. www.structech.us/SHEARWALL-Rev1.ppt
[16].

www.iitk.ac.in/.../SeismicBehaviour_Design\&DetailingofShea rWalls-...

[17]. www.wisegeek.com/what-is-a-shear-wall.htm 